\title{
Psychometric Testing of the Social Support Survey on a Taiwanese Sample
}

\author{
Yea-Ing Lotus Shyu $\boldsymbol{\nabla}$ Woung-Ru Tang $\boldsymbol{\nabla}$ Jersey Liang $\boldsymbol{\nabla}$ Li-Jen Weng
}

\section{Editor's Note}

Materials documenting the review process for this article are posted at http://www.nursing-research-editor.com.

Background: The Medical Outcomes Study Social Support Survey (MOS-SS) is a multidimensional, self-administered instrument used to assess various functional dimensions of social support. Less is known regarding its value for a Chinese population.

- Objective: The aim of this study was to assess the psychometric properties of the MOS-SS on an adult sample in Taiwan.

Methods: A total of 265 adult family caregivers of patients with cancer from four different hospitals in the north, middle, and south regions of Taiwan completed the MOS-SS.

- Results: In the original five-factor model, unsatisfactory item discriminant validity was found in almost half of the items; the item-own subscale correlation was lower than the item-other subscale correlation. A two-factor model accounting for $68.98 \%$ of the variance was found using exploratory factor analysis. The first factor (emotional support) accounted for $62.28 \%$ of the total variance, whereas the second factor (tangible support) accounted for $6.7 \%$; the interfactor correlation was .71. The two-factor model seemed to have satisfactory reliability and validity and better discrimination between different subscales than did the original five-factor model.

Discussion: Good reliability and validity were demonstrated in the MOS-SS when applied to an adult sample in Taiwan. A two-factor model, instead of a five-factor model as found in the Western countries, was found for this sample. Confirmation of the two-factor model and exploration of the two-factor model in related concepts are suggested for future studies.

Key Words: emotional social support · factor analysis . tangible social support

$\mathbf{T}$ he importance of social support when protecting individuals from major illness and stressful situa- tions has been addressed extensively (Berkman \& Syme, 1979; Blazer, 1982; House, Robbins, \& Metzner, 1982; Sherbourne, 1988). Therefore, assessment of social support is important for understanding and predicting the health outcomes of an individual. The construct of social support is a metaconcept, and methods used to assess social support vary because of different conceptualizations of it (Donald \& Ware, 1984; House \& Kahn, 1985; Hutchison, 1999; O'Reilly, 1988). The concept has been studied primarily from three perspectives: network structure, support functions, and the nature of relationships (Hutchison, 1999). The network structure refers to the existence and the quantity of interpersonal relationships and the interconnectedness of social relationships. This approach may measure concepts that are unrelated to support, such as the need for contact or how busy a person is (Sherbourne \& Stewart, 1991). In recent years, the perceived availability of a functional component of social support has been emphasized (Cohen \& Wills, 1985; House \& Kahn, 1985; Sherbourne \& Stewart, 1991). This approach emphasizes the functions of interpersonal relationships, such as emotional support, instrumental support, informational support, social companionship, and appraisal support (Cohen \& Hoberman, 1983; House, 1981; Wills, 1985).

The Medical Outcomes Study Social Support Survey (MOS-SS) is a multidimensional, self-administered instrument that addresses all five functions of an interpersonal relationship to assess the various functional dimensions of

Yea-Ing Lotus Shyu, is Professor, School of Nursing, Center for Gerontological Research, Chang Gung University, Taoyuan, Taiwan.

Woung-Ru Tang, is Assistant Professor, Graduate Institute of Nursing, Chang Gung University, Taoyuan, Taiwan.

Jersey Liang, is Professor, School of Public Health, and Senior Research Scientist, Institute of Gerontology, University of Michigan, Ann Arbor.

Li-Jen Weng, is Professor, Department and Graduate Institute of Psychology, National Taiwan University, Taipei, Taiwan. 
social support, including emotional, informational, tangible, affectionate, and positive interaction (Sherbourne \& Stewart, 1991). In the study of Sherbourne and Stewart (1991), the reliability and validity of the survey in patients with chronic illness in the United States were established. It has also been used to measure social support for patients with breast carcinoma and patients who received a bone marrow transplant in Western countries, and it demonstrated good reliability (Heinonen et al., 2001; Kornblith et al., 2003). However, little is known on the validity and reliability of the MOS-SS for a Chinese population. Taiwan differs substantially from Western countries in healthcare system, clinical practice, case mix, culture, and social organization. For example, unlike the United States, Taiwan has national health insurance, and individuals have the choice to visit clinics in a medical center without referrals. In addition, in Taiwan, approximately $90 \%$ of dependent elderly persons are cared for by their family members (Directorate-General of Budget, Accounting and Statistics, Executive Yuan and Ministry of Interior, Republic of China, 1997). In the Chinese culture, devotion to parents, social orientation, and interdependence are emphasized, whereas in Western culture, autonomy and independence are valued and oversacrifice for parents is not encouraged (Dai \& Dimond, 1998). These differences may cause a different presentation of social support between Taiwan and Western countries.

Because psychometric properties are sample dependent, the performance of a measure in a specific application is especially important (McHorney, Ware, Lu, \& Sherbourne, 1994). For a further comparison of the nature and role of social support in health outcomes across nations and cultures, it is necessary to establish the validity and reliability of the MOS-SS among Taiwanese adults. The purpose of this study is, therefore, to describe the psychometric properties of the Taiwan version of the MOS-SS on an adult population. Formal psychometric tests of the assumptions underlying the item scoring and the construction of multiitem scales were conducted. Then, exploratory factor analysis was used to explore the dimensionality of the MOS-SS in a Taiwanese sample. After a two-factor approach was identified and proposed in place of the original five-factor solution, the two-factor approach received further psychometric and construct validity testing in this sample.

\section{Methods}

\section{Study Setting and Sample}

The sample $(N=265)$ consisted of the family caregivers of patients with cancer from four different hospitals in the north, middle, and south regions of Taiwan. Inclusion criteria were (a) being the primary family caregivers for patients with cancer and (b) being 18 years of age or older.

\section{Procedures}

Human subjects approval was obtained from the medical centers. The primary family caregivers were contacted and invited to participate in the study after the care recipient had received either palliative care or active treatment for at least 1 week in a hospital. Data were collected at hospitals through self-reported questionnaires.

\section{Instruments}

MOS Social Support Survey A total of 19 functional support items hypothesized to measure emotional, informational, tangible, and affectionate support and positive social interaction were developed to assess the perceptions of the availability of different functional aspects of support (Sherbourne \& Stewart, 1991). Emotional support contains four items measuring the expression of positive affect, empathetic understanding, and encouragement feeling expressions. Information support contains four items measuring the provisions of advice, information, guidance, or feedback. Tangible support contains four items measuring the offering of material aid or behavioral assistance. Affectionate support contains three items measuring the expressions of love and affection. Positive social interaction contains four items measuring the availability of other persons to do fun things with you. For each item, the respondents were asked to indicate how often each support was available to them if they needed it. Response options were none of the time (1), a little of the time (2), some of the time (3), most of the time (4), and all of the time (5). For each scale, simple algebraic sums were computed, and then the raw scale scores were transformed into a scale of 0 to 100 . The higher the score, the better the perception of social support. The reliability and validity of the measure in an American population of adults with chronic illness have been established (Sherbourne \& Stewart, 1991).

The Taiwan version of the MOS-SS was identical to the original version. Translation and backtranslation were used to ensure the language equivalence of the original language and the target language. Conceptual equivalence is extremely important when translating instruments (Maneesriwongul, 2004; Willgerodt, Kataoka-Yahiro, Kim, \& Ceria, 2005). Translation and backtranslation of the MOS-SS was performed by two bilingual and Englishspeaking experts (Brislin, 1970; Jones, 1987). After translation and backtranslation were done, the research team checked both language equivalence and conceptual equivalence.

Spirituality (Validity Variable) The Spiritual Well-Being Scale (SWBS; Paloutzian \& Ellison, 1982) was used to measure spirituality and to assess the construct validity of the MOS-SS by using hypothesis testing. Spirituality has a positive association to social support and a negative association to loneliness (Pace \& Stables, 1997). The reliability and validity of the SWBS have been tested (Coleman \& Holzemer, 1999; Fernsler, Klemm, \& Miller, 1999; Isaia, Parker, \& Murrow, 1999; Mickley \& Soeken, 1993), and national norms were obtained on the SWBS across a variety of studies (Bufford, Paloutzian, \& Ellison, 1991). The Chinese version of the SWBS has been tested on a population with lung cancer and has been proved to retain good psychometric properties (Su, 2002). In this study, Cronbach's $\alpha$ values of .88 were found for the sample of caregivers of patients with cancer.

General Health Status (Validity Variable) A single-item indicator that is used commonly (Hays, Kallich, Mapes, Coons, \& Carter, 1994; Hays, Sherbourne, \& Mazel, 1993; Jenkinson, Lawrence, McWhinnie, \& Gordon, 1995; Tang, 2001) to measure an individual's general health status was 
selected to measure the general health status. Internal consistency reliability is unavailable for this single-item indicator. However, validity was supported by its association with pain intensity $(r=.56, p<.001)$, physical performance status $(r=.53, p<.001)$, and physical wellbeing $(r=-.61, p<.001)$ in a population of caregivers taking care of terminally ill patients (Tang, 2001).

\section{Data Analysis}

Means, standard deviations, item-scale correlations, Cronbach's $\alpha$, and interscale correlations were used to examine whether the scores satisfied the scaling assumptions of the MOS-SS. The convergent validity of items in a hypothesized scale of the MOS-SS was examined by item-own scale correlation. The divergent validity of the items in relation to other scales that one expects to be independent was examined by item-other scale correlation. The Pearson product-moment correlation of the MOS-SS with spirituality and general health status was used to test the hypothesized relationship between social support and related concepts (Crocker \& Algina, 1986). It was expected that a significant positive correlation existed between spirituality and social support (Koenig, George, \& Titus, 2004) and between general health and social support (Israel, Farquhar, Schulz, James, \& Parker, 2002; Westaway, Seager, Rheeder, \& Van Zyl, 2005).

Exploratory factor analysis was used to examine and explore the dimensionality of the social support measure. All of the data were coded and entered into a computer and analyzed by SPSS 10.0 .

\section{Results}

Psychometric Analysis of the Original Scaling Assumptions

The characteristics of the sample are presented in Table 1. Approximately $60.2 \%$ were women, with an average age of 42.14 years $(S D=13.62)$. Most $(68.8 \%)$ were married, and approximately $70.1 \%$ had a high school education or less.

The percentage of missing data, response-option frequency distribution, mean, standard deviation, and skewness of each item were grouped under each hypothesized subscale. The missing-value rates ranged from $0 \%$ to $1.9 \%$. Item response-option frequency distributions were relatively symmetrical, and the skewness was between -1.0 and +1.0 for all items. Items had similar standard deviations, which supports the scaling assumptions on the equal item variance when measuring the same concept.

The item-subscale correlations of the original subscales are presented in Table 2 (Sherbourne \& Stewart, 1991). The correlations between each item and its hypothesized subscale were all above .6 , indicating that most items met the scaling assumption of internal consistency. However, unsatisfactory item discriminant validity was found in almost half of the items (Items 6, 7, 8, 9, 10, 13, 17, 18, and 20), with itemown subscale correlation being lower than item-other subscale correlation. The item-other subscale association ranged from .43 to .84 , with approximately $45 \%$ being .7 or .8 .

The ranges of scores for the original five dimensions of social support demonstrated good variability (Table 3 ). The skewness of all scales was within the recommended range of -1.0 to +1.0 . Floor effects around $0.8 \%$ to $1.5 \%$ and ceiling effects around $7.9 \%$ to $13.6 \%$ were found.

\begin{tabular}{|c|c|}
\hline & $n(\%)$ \\
\hline \multicolumn{2}{|l|}{ Gender } \\
\hline Male & $105(39.6)$ \\
\hline Female & $160(60.4)$ \\
\hline Age $(M \pm S D)$ & $42.14 \pm 13.62$ \\
\hline \multicolumn{2}{|l|}{ Marital status } \\
\hline Single & $59(22.3)$ \\
\hline Married & $183(69.1)$ \\
\hline Not married but lives with a partner & $3(1.1)$ \\
\hline Separated & $3(1.1)$ \\
\hline Widowed & $10(3.8)$ \\
\hline Divorced & $7(2.6)$ \\
\hline \multicolumn{2}{|l|}{ Educational background } \\
\hline Illiterate & $20(7.5)$ \\
\hline Primary school & $38(14.3)$ \\
\hline High school & $128(48.3)$ \\
\hline College or above & $79(29.8)$ \\
\hline
\end{tabular}

\section{Exploratory Factor Analysis}

Exploratory factor analysis is used primarily as a means of exploring the underlying factor structure without prior specification of the number of factors (Bollen, 1989). Because of the close differences between item-own subscale and item-other subscale correlations for items in the MOSSS, the exploratory factor analysis was used first to explore and interpret the underlying factors. Two initial eigenvalues from a principal components analysis were found to be greater than 1 (11.83 and 1.27). A scree plot is presented in Figure 1. Therefore, a two-factor solution that accounted for $68.98 \%$ of the variance was selected (Table 4). After using the common factor model, followed by a varimax rotation, patterns of high and low loadings across variables were examined to define the factor labels. Items originally hypothesized to be in the subscale of tangible support, including the availability of someone to help if the patient is confined to a bed, to take one to a doctor, to prepare meals, and to help with daily chores, loaded high on the second factor. The remaining items of the MOS-SS were found to load high on the first factor. The first factor (emotional support) accounted for $62.28 \%$ of the total variance, whereas the second factor (tangible support) accounted for $6.7 \%$.

The interfactor correlation was .71; as perceived availability of emotional support improved, so did tangible support. As further evidence of the internal consistency of the two-factor solution, the Cronbach's $\alpha$ coefficient was found to be .97 for emotional support and .85 for tangible support.

\section{Psychometric Testing and Construct Validity for the Two-factor Model}

The item-subscale correlation of the two-factor model was examined further (Table 5). The correlations between an item and its hypothesized subscale ranged from .60 to .85 , 
TABLE 2. The Pearson Item-subscale Correlations Between Fach Item and the Original Hypothesized Subscale

Social Support Scale

\begin{tabular}{lcccccc}
\cline { 2 - 6 } Scale & Item & Tangible & Emotional & Affective & Positive Social Interaction & Informational \\
\hline Tangible & 2 & .601 & .488 & .425 & .445 & .546 \\
& 5 & .668 & .589 & .598 & .546 & .605 \\
Emotional & 12 & .708 & .542 & .497 & .591 & .528 \\
& 15 & .733 & .600 & .527 & .772 & .541 \\
& 9 & .602 & .774 & .797 & .750 & .801 \\
Affective & 16 & .594 & .834 & .710 & .786 & .741 \\
& 17 & .592 & .833 & .699 & .794 & .838 \\
Positive social interaction & 19 & .594 & .820 & .742 & .739 & .755 \\
& 6 & .673 & .753 & .679 & .718 & .726 \\
& 10 & .455 & .689 & .677 & .664 & .628 \\
Informational & 20 & .524 & .700 & .632 & .771 & .636 \\
& 7 & .599 & .751 & .775 & .780 & .751 \\
& 11 & .534 & .757 & .734 & .751 & .705 \\
& 14 & .593 & .735 & .632 & .798 & .727 \\
& 18 & .572 & .816 & .736 & .677 & .735 \\
& 3 & .618 & .737 & .638 & .635 & .745 \\
\end{tabular}

Note. Values in bold are corrected correlations for that item, and its hypothesized scale that excludes the item was calculated. For all values, $p<.01$.

and item-other subscale correlation ranged from .46 to 67. Discrepancies among items in different subscales seemed to be more distinguishable than in the original hypothesized subscales (Sherbourne \& Stewart, 1991).

TABLE 3. Missing Percentage Floor and

Ceiling Effects, Means, Standard Deviations, and Skews of Fach Subscale in the MOS-sS $(\mathbf{N}=\mathbf{2 6 5})$

\begin{tabular}{lcrrrr} 
& \multicolumn{2}{c}{ Percentage } & & & \\
\cline { 2 - 5 } Scale & $\begin{array}{c}\text { Floor } \\
\text { Effect }\end{array}$ & $\begin{array}{c}\text { Ceiling } \\
\text { Effect }\end{array}$ & Mean & SD & Skew \\
\hline Tangible & 1.1 & 11.3 & 67.64 & 22.58 & -0.566 \\
Emotional & 1.5 & 9.8 & 61.37 & 24.02 & -0.297 \\
Affective & 1.1 & 13.6 & 65.69 & 23.72 & -0.373 \\
Positive & 0.8 & 11.7 & 63.42 & 23.45 & -0.273 \\
$\quad$ social & & & & & \\
$\quad$ interaction & & & & & \\
Informational & 1.1 & 7.9 & 65.09 & 21.01 & -0.385 \\
\hline
\end{tabular}

Note. MOS-SS $=$ Medical Outcomes Study Social Support Survey.
The variability, skewness, and floor and ceiling effects of the two-factor model are similar to those of the original hypothesized five-factor model (Sherbourne \& Stewart, 1991). The mean $\pm S D$ of scores for the two-factor model were $67.64 \pm 22.58$ for tangible support and $63.69 \pm 21.55$ for emotional support. The skewness was -0.566 for tangible support and -0.306 for emotional support; both

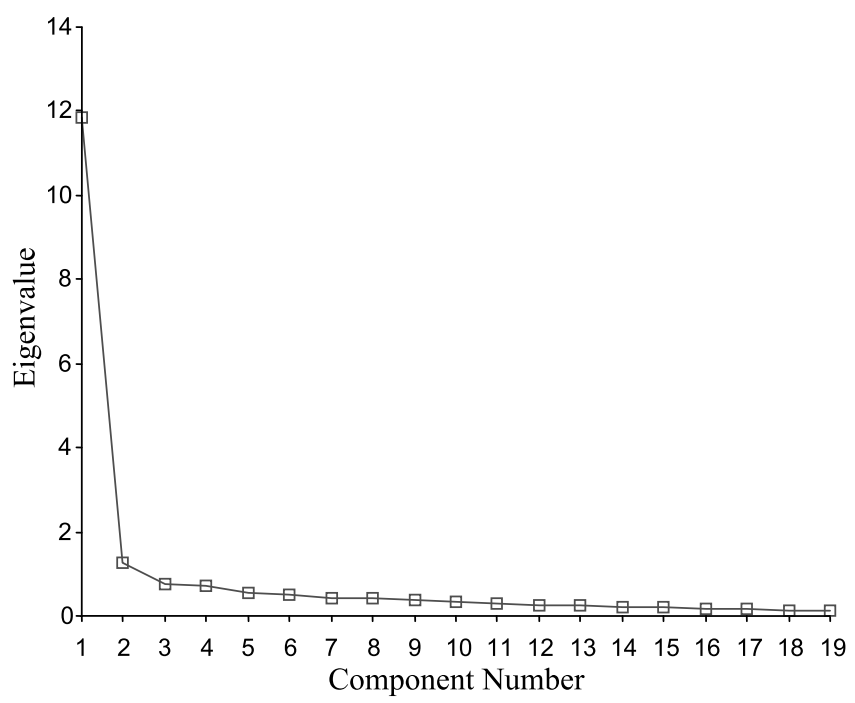

FIGURE 1. Scree plot. 
TABLE 4. Rotated Factor Loadings for MOS Social Support Scale

\begin{tabular}{llc} 
& \multicolumn{2}{l}{ Factor Loadings } \\
\cline { 2 - 3 } Items & 1 & 2 \\
\hline Emotion support subscale & & \\
$\quad$ Turn to for suggestions (17) & .808 & \\
Do something enjoyable with (18) & .783 & \\
Confide in (9) & .783 & \\
Understand your problems (19) & .775 & \\
Give advice you really want (13) & .766 & \\
Give you information (8) & .764 & \\
Get together for relaxation (11) & .761 & \\
Share worries with (16) & .734 & \\
Have a good time with (7) & .721 & \\
Hugs you (10) & .715 & \\
Do things (14) & .682 & \\
Gives you good advice (4) & .659 & \\
Listens to you (3) & .655 & \\
Show love and affection (6) & .632 & \\
Loves you (20) & .629 & .6935 \\
Tangible support subscale & & \\
Helps with daily chores (15) & & .758 \\
Prepares meals (12) & & \\
Takes you to doctor (5) & & \\
Helps if confined to bed (2) & & \\
Eigenvalue & & \\
\% Variance & & \\
Cumulative variance & & \\
\hline
\end{tabular}

Note. MOS $=$ Medical Outcomes Study.

were within the recommended -1.0 to +1.0 range. Floor effects were $1.1 \%$ for tangible support and $0.4 \%$ for emotional support, and the ceiling effects were $11.3 \%$ for tangible support and 3.4\% for emotional support.

In terms of construct validity, spirituality was found to have a positive significant association with emotional $(r=$ $.35 ; p<.01)$ and tangible $(r=.19 ; p<.01)$ support. The general health status was related positively both to emotional $(r=.26 ; p=.000)$ and tangible $(r=.21 ; p=.001)$ support.

In summary, the two-factor model of the MOS-SS seemed to improve the differentiation between the subscales of the five-factor model. At the same time, the variability, skewness, and floor and ceiling effects for each subscale were similar to those of the original five-factor model. Satisfactory reliability and validity were found for the twofactor model.

\section{Discussion}

Information on the psychometric properties of the MOS-SS in an adult sample in Taiwan is provided in this study. The evaluation of scaling assumptions and score reliability is very important when applied to a particular sample. In this study, good evidence was demonstrated with the MOS-SS to support the scaling assumptions when administered to an adult sample in Taiwan. The relatively high variance and symmetric distributions of item-response frequencies of all items supported the ability of the items in this scale in measuring the variability of the phenomena (DeVellis, 1991). For the original hypothesized five-factor model, the scaling assumption is supported by equal item variance in measuring the same concept and by the high Pearson item-scale correlation between each item and the hypothesized subscale (DeVellis, 1991). Good variability, symmetric distribution, and acceptable floor and ceiling effects of the subscale score were also found for each original hypothesized subscale. However, because the item-own subscale correlations were lower than the item-other subscale correlations for almost half of the items, item discriminant validity was not achieved for the original hypothesized five subscales.

In terms of the factor structure of the MOS-SS, the twofactor model, with factor loadings from .63 to .81 and an interfactor correlation of .71, was selected. According to the comparison of the psychometric testing results of the twofactor model with the original five-factor model, the theoretical structure of factors containing tangible and emotional

TABLE 5. The Pearson Item-subscale

Correlation Between Fach Item and the

Two-factor Subscale

\begin{tabular}{|c|c|c|c|}
\hline \multirow[b]{2}{*}{ Scale } & \multirow[b]{2}{*}{ Item } & \multicolumn{2}{|c|}{ Two-factor Solution } \\
\hline & & Tangible & Emotional \\
\hline \multirow[t]{4}{*}{ Tangible } & 2 & .601 & .504 \\
\hline & 5 & .668 & .625 \\
\hline & 12 & .708 & .572 \\
\hline & 15 & .733 & .608 \\
\hline \multirow[t]{15}{*}{ Emotional } & 3 & .618 & .750 \\
\hline & 4 & .552 & .717 \\
\hline & 6 & .673 & .785 \\
\hline & 7 & .599 & .818 \\
\hline & 8 & .590 & .814 \\
\hline & 9 & .602 & .847 \\
\hline & 10 & .455 & .726 \\
\hline & 11 & .534 & .798 \\
\hline & 13 & .582 & .817 \\
\hline & 14 & .593 & .765 \\
\hline & 16 & .594 & .809 \\
\hline & 17 & .592 & .849 \\
\hline & 18 & .572 & .834 \\
\hline & 19 & .594 & .835 \\
\hline & 20 & .524 & .704 \\
\hline
\end{tabular}

Note. Values in bold are corrected correlations for that item, and its hypothesized scale that excludes the item was calculated. For all values, $p<.01$. 
social supports seemed to be more empirically distinguishable, with similar validity and reliability in a Taiwanese sample. The social support components have been found to be correlated too highly to be differentiated empirically (Norbeck, Lindsey, \& Carrieri, 1981; House \& Kahn, 1985; Sherbourne \& Stewart, 1991). As stated by Sherbourne and Stewart (1991), emotional and informational support indicated support communication, and they suspected that what they had labeled affection is really emotional support. In a recent article, Stewart, Teno, Patrick, and Lynn (1999) specified tangible and emotional support as social support in a conceptual model for factors affecting the quality and length of life of dying patients and their families. However, positive interaction, affection, and informational support were not listed specifically in the model. Recently, Westaway et al. (2005) extracted two social support factors, socioemotional support and tangible support, that accounted for $78.9 \%$ of the variance from MOS-SS in a sample of Black outpatients with diabetes mellitus.

For the two-factor model in this Taiwanese sample, equal item variance in measuring the same concept was supported by a similar standard deviation of all items. Internal consistency was supported by Cronbach's $\alpha$ coefficients of above .70 for both subscales. The scaling assumption of internal consistency of each subscale is supported by the high Pearson item-scale correlation between each item and the hypothesized subscale (DeVellis, 1991). Good variability, symmetric distribution, and acceptable floor and ceiling effects of the subscale score were found for both subscales. Thus, we can conclude that the reliability of the two-factor MOS-SS was established in an adult sample in Taiwan.

The consistently higher correlation between an item and its own subscale than between an item and other subscale supports the discriminant validity of the twofactor model. The construct validity of the two-factor MOS-SS was supported by research showing that general health was associated positively with both emotional and tangible social support (Israel et al., 2002; Sherbourne \& Stewart, 1991; Westaway et al., 2005). As in this study, the association between general health and different dimension of social support was around .20 (Sherbourne \& Stewart, 1991). In the study of Koenig et al. (2004), selfrated spirituality was associated significantly with social support at $r=.19$. Spirituality was associated positively with emotional social support at $r=.35$ and tangible social support at $r=.19$ in this study.

In summary, these findings provide a preliminary basis for the use of the MOS-SS in a Taiwanese sample. The small convenience sample limits the generalizability of the results. The fact that all the participants were family caregivers might have influenced their perception of social support, and therefore, careful considerations might need to be paid to the generalization of the study results before applying them to noncaregiver adult populations. A more representative and larger sample using confirmatory factor analysis to confirm the factor structure and using structural equation modeling to further explore the underlying concepts in related concepts is suggested for future studies.
Whether the two-factor model is culture specific should also be studied. In its current form, the two-factor MOS-SS model demonstrated good psychometric properties as applied to an adult sample in Taiwan. The study results may be applicable to other countries with Chinese populations and can provide a reference for instrumentation of social support measures in other Chinese populations.

Accepted for publication June 12, 2006.

This work was funded by the National Health Research Institute and Chang Gung Memorial Hospital, Taiwan.

Corresponding author: Yea-Ing Lotus Shyu, School of Nursing, Center for Gerontological Research, Chang Gung University, Taoyuan, Taiwan (e-mail:yeaing@mail.cgu.edu.tw).

\section{References}

Berkman, L. F., \& Syme, S. L. (1979). Social networks, host resistance and mortality: A nine-year follow-up study of Alameda County residents. American Journal of Epidemiology, 109, 186-204.

Blazer, D. G. (1982). Social support and mortality in an elderly community population. American Journal of Epidemiology, $115,684-694$.

Bollen, K. A. (1989). Structural equations with latent variables. New York: Wiley.

Brislin, R. W. (1970). Back-translation for cross-culture research. Journal of Cross Cultural Psychology, 1, 185-216.

Bufford, R. K., Paloutzian, R. F., \& Ellison, C. W. (1991). Norms for the Spiritual Well-Being Scale. Journal of Psychology and Theology, 19, 56-70.

Cohen, S., \& Hoberman, H. (1983). Positive events and social supports as buffers of life change stress. Journal of Applied Social Psychology, 13, 99-125.

Cohen, S., \& Wills, T. A. (1985). Stress, social support, and the buffering hypothesis. Psychological Bulletin, 98, 310-357.

Coleman, C. L., \& Holzemer, W. L. (1999). Spirituality, psychological well-being, and HIV symptoms for African Americans living with HIV disease. The Journal of the Association of Nurses in AIDS Care, 10, 42-50.

Crocker, L., \& Algina, J. (1986). Introduction to classical and modern test theory. New York: Holt, Rinehart \& Winston.

Dai, Y. T., \& Dimond, M. F. (1998). Filial piety. A cross-cultural comparison and its implications for the well-being of older parents. Journal of Gerontological Nursing, 24(3), 13-18.

DeVellis, R. F. (1991). Scale development: Theory and applications. Newbury Park, CA: Sage.

Directorate-General of Budget, Accounting and Statistics, Executive Yuan and Ministry of Interior, Republic of China. (1997). Report on the old status survey: Taiwan area, Republic of China. Taipei, Taiwan: Author.

Donald, C. A., \& Ware, J. E., Jr. (1984). The measurement of social support. In R. Greenley (Ed.), Research in community and mental health (pp. 325-370). Greenwich, CT: JAI Press.

Fernsler, J. I., Klemm, P., \& Miller, M. A. (1999). Spiritual wellbeing and demands of illness in people with colorectal cancer. Cancer Nursing, 22(2), 134-140.

Hays, R. D., Kallich, J. D., Mapes, D. L., Coons, S. J., \& Carter, W. B. (1994). Development of the kidney disease quality of life (KDQOL) instrument. Quality of Life Research, 3, 329-338.

Hays, R. D., Sherbourne, C. D., \& Mazel, R. M. (1993). The RAND 36-Item Health Survey 1.0. Health Economics, 2(3), 217-227.

Heinonen, H., Volin, L., Uutela, A., Zevon, M., Barrick, C., \& Ruutu, T. (2001). Quality of life and factors related to perceived 
satisfaction with quality of life after allogeneic bone marrow transplantation. Annals of Hematology, 80(3), 137-143.

House, J. S. (1981). Work, stress and social support. Reading, MA: Addison-Wesley.

House, J. S., \& Kahn, R. L. (1985). Measures and concepts of social support. In S. Cohen \& L. Syme (Eds.), Social support and health (pp. 83-108). San Francisco: Academic Press.

House, J. S., Robbins, C., \& Metzner, H. L. (1982). The association of social relationships and activities with mortality: Prospective evidence from the Tecumseh Community Health Study. American Journal of Epidemiology, 116, 123-140.

Hutchison, C. (1999). Social support: Factors to consider when designing studies that measure social support. Journal of Advanced Nursing, 29, 1520-1526.

Isaia, D., Parker, V., \& Murrow, E. (1999). Spiritual well-being among older adults. Journal of Gerontological Nursing, 25(8), $15-21$.

Israel, B. A., Farquhar, S. A., Schulz, A. J., James, S. A., \& Parker, E. A. (2002). The relationship between social support, stress, and health among women on Detroit's East Side. Health Education \& Behavior, 29(3), 342-360.

Jenkinson, C., Lawrence, K., McWhinnie, D., \& Gordon, J. (1995). Sensitivity to change of health status measures in a randomized controlled trial: Comparison of the COOP charts and the SF-36. Quality of Life Research, 4(1), 47-52.

Jones, E. (1987). Translation of quantitative measures for use in cross-culture research. Nursing Research, 36, 324-327.

Koenig, H. G., George, L. K., \& Titus, P. (2004). Religion, spirituality, and health in medically ill hospitalized older patients. Journal of the American Geriatrics Society, 52, 554-562.

Kornblith, A. B., Herndon, J. E., 2nd, Weiss, R. B., Zhang, C., Zuckerman, E. L., Rosenberg, S., et al. (2003). Long-term adjustment of survivors of early-stage breast carcinoma, 20 years after adjuvant chemotherapy. Cancer, 98, 679-689.

Maneesriwongul, W. (2004). Instrument translation process: A methods review. Methodological Issues in Nursing Research, 48(2), 175-186.

McHorney, C. A., Ware, J. E., Jr., Lu, J. F., \& Sherbourne, C. D. (1994). The MOS 36-item Short-Form Health Survey (SF-36): III. Tests of data quality, scaling assumptions, and reliability across diverse patient groups. Medical Care, 32(1), 40-66.
Mickley, J., \& Soeken, K. (1993). Religiousness and hope in Hispanic- and Anglo-American women with breast cancer. Oncology Nursing Forum, 20, 1171-1177.

Norbeck, J. S., Lindsey, A. M., \& Carrieri, V. L. (1981). The development of an instrument to measure social support. Nursing Research, 30(5), 264-269.

O'Reilly, P. (1988). Methodological issues in social support and social research. Social Science \&o Medicine, 26, 863-873.

Pace, J. C., \& Stables, J. L. (1997). Correlates of spiritual wellbeing in terminally ill persons with AIDS and terminally ill persons with cancer. The Journal of the Association of Nurses in AIDS Care, 8(6), 31-42.

Paloutzian, R. F., \& Ellison, C. W. (1982). Loneliness, spiritual well-being, and quality of life. In L. A. Peplau (Ed.), Loneliness: A sourcebook of current theory, research and theory (pp. 224-237). New York: Wiley.

Sherbourne, C. D. (1988). The role of social support and life stress events in use of mental health services. Social Science \& Medicine, 27, 1393-1400.

Sherbourne, C. D., \& Stewart, A. L. (1991). The MOS Social Support Survey. Social Science \& Medicine, 32, 705-714.

Stewart, A. L., Teno, J., Patrick, D. L., \& Lynn, J. (1999). The concept of quality of life of dying persons in the context of health care. Journal of Pain and Symptom Management, 17(2), 93-108.

Su, S. F. (2002). Psychometric assessment of Spiritual Well-Being Scale in Taiwan. Unpublished master's thesis, Chang Gung University, Taoyuan, Taiwan.

Tang, W. R. (2001). Terminally ill patients and their caregivers' quality of life. Dissertation Abstracts International, 62(04), 1810B (UMI No. 3013516).

Westaway, M. S., Seager, J. R., Rheeder, P., \& Van Zyl, D. G. (2005). The effects of social support on health, well-being and management of diabetes mellitus: A Black South African perspective. Ethnicity \& Health, 10(1), 73-89.

Willgerodt, M. A., Kataoka-Yahiro, M., Kim, E., Ceria, C. (2005). Issues of instrument translation in research on Asian immigrant populations. Journal of Professional Nursing, 21(4), 231-239.

Wills, T. A. (1985). Supportive functions of interpersonal relationships. In S. Cohen \& S. L. Syme (Eds.), Social support and health (pp. 61-82). Orlando, FL: Academic Press. 